\title{
Self-Propelled Particles with Velocity Reversals and Ferromagnetic Alignment: Active Matter Class with Second-Order Transition to Quasi-Long-Range Polar Order
}

\author{
B. Mahault, ${ }^{1}$ X.-c. Jiang, ${ }^{2}$ E. Bertin, ${ }^{3}$ Y.-q. Ma, ${ }^{4,2}$ A. Patelli, ${ }^{1}$ X.-q. Shi, ${ }^{2,1}$ and H. Chate $e^{1,5,6}$ \\ ${ }^{1}$ Service de Physique de l'Etat Condensé, CEA, CNRS, Université Paris-Saclay, CEA-Saclay, 91191 Gif-sur-Yvette, France \\ ${ }^{2}$ Center for Soft Condensed Matter Physics and Interdisciplinary Research, Soochow University, Suzhou 215006, China \\ ${ }^{3}$ LIPHY, Université Grenoble Alpes and CNRS, F-38000 Grenoble, France \\ ${ }^{4}$ National Laboratory of Solid State Microstructures and Department of Physics, Nanjing University, Nanjing 210093, China \\ ${ }^{5}$ Beijing Computational Science Research Center, Beijing 100094, China \\ ${ }^{6}$ Sorbonne Université, CNRS, Laboratoire de Physique Théorique de la Matière Condensée, 75005 Paris, France
}

(Received 7 March 2018; published 20 June 2018)

\begin{abstract}
We introduce and study in two dimensions a new class of dry, aligning active matter that exhibits a direct transition to orientational order, without the phase-separation phenomenology usually observed in this context. Characterized by self-propelled particles with velocity reversals and a ferromagnetic alignment of polarities, systems in this class display quasi-long-range polar order with continuously varying scaling exponents, yet a numerical study of the transition leads to conclude that it does not belong to the Berezinskii-Kosterlitz-Thouless universality class but is best described as a standard critical point with an algebraic divergence of correlations. We rationalize these findings by showing that the interplay between order and density changes the role of defects.
\end{abstract}

DOI: 10.1103/PhysRevLett.120.258002

Dry active matter refers to systems of mobile agents for which the surrounding fluid can be neglected $[1,2]$. A wealth of spectacular phenomena has been uncovered, particularly in situations where some local alignment of velocities arises, such as animal groups $[3,4]$, bacteria and cells crawling on a substrate [5-7], motility assays [8], and vertically shaken granular particles [9-12].

We now have a satisfactory theoretical understanding of dry, aligning active matter in the dilute limit, thanks to a series of works on particle, hydrodynamic, and kinetic levels [13-42]. In these systems, the dominating interaction, local alignment, is in competition with noise, and it can lead to orientationally ordered phases that are endowed with generic long-range correlations and anomalous fluctuations [14-16,20,22,25,26,36]. The emergence of order is not a direct, continuous phase transition but occurs via phase separation between a disordered gas and an ordered liquid separated by a coexistence phase $[34,38,40]$. Genuine nontrivial critical behavior has only been found when long-range interactions are present, brought by nonmetric, "topological" neighbors $[43,44]$ or by imposing some incompressibility condition $[45,46]$.

Published by the American Physical Society under the terms of the Creative Commons Attribution 4.0 International license. Further distribution of this work must maintain attribution to the author(s) and the published article's title, journal citation, and DOI.
Vicsek-style models, which consist of constant-speed point particles that locally align their velocities in competition with some noise, have been instrumental in this success. Their simplicity allows for both in-depth numerical study and the controlled derivation of hydrodynamic theories [19,21,23,27-31,37]. Each of the three main classes of dry, aligning active matter studied thus far possesses a Vicsek-style representative. Polar particles with ferromagnetic alignment-the case of the original Vicsek model [13] — give rise to true long-range polar order $[14,15,32]$ and a coexistence phase made of quantized traveling bands [17,22,35,39,40,47]. Nematic alignment leads to global nematic order and a chaotic coexistence phase mediated by unstable nematic bands [20,33,36,41]. This case is customarily divided into two classes [1,2] since, for polar particles, nematic order seems to be longrange $[7,25,42]$, whereas for a finite velocity-reversal rate it is only quasi-long-range [20,36].

In this Letter we show that self-propelled particles with velocity reversals and local ferromagnetic alignment exhibit novel collective properties and, in particular, a continuous transition to order. It is rather obvious that this case cannot lead to even local orientational order if one considers only the restricted Vicsek setting where a particle's polarity is simply given by its velocity [48]. Here, we relax this "Vicsek constraint" by conferring particles a polarity that they align with that of their neighbors while they move either along or against it [49]. Using kinetic and hydrodynamic-level descriptions derived from the microscopic model, we show that the 
analytic structure of this problem is qualitatively different from that of the three other classes. In particular, it is deprived from the generic linear instability at the root of the liquid-gas phase separation scenario. Particle-level simulations confirm this: the emerging polar order is only quasilong-range with continuously varying scaling exponents, while showing giant number fluctuations. Thus, this case possesses many of the properties of the (equilibrium) $X Y$ model. Yet, surprisingly, a numerical study of the continuous ordering transition leads us to conclude that it does not belong to the Berezinskii-Kosterlitz-Thouless universality class [50-52] characteristic of the $X Y$ model, but that it is best described as a standard critical point with an algebraic divergence of correlations. We rationalize these findings by showing that the coupling between order and density deprives defects from their usual role.

We first define our "Vicsek-shake" model. We restrict ourselves to two space dimensions and the square domains of linear size $L$ with periodic boundary conditions. The velocity $\mathbf{v}_{i}$ of particle $i$ is given by $\mathbf{v}_{i}= \pm v_{0} \mathbf{p}_{i}$, where the unit vector $\mathbf{p}_{i}$ is the particle's intrinsic polarity, and the sign is changed with probability $\alpha$ at each unit time step. Positions and polarities are governed by

$$
\begin{aligned}
& \mathbf{r}_{i}(t+1)=\mathbf{r}_{i}(t)+\mathbf{v}_{i}(t+1), \\
& \mathbf{p}_{i}(t+1)=\left(\mathcal{R}_{\eta} \circ \Pi\right)\left\langle\mathbf{p}_{j}(t)\right\rangle_{j \in \partial_{i}},
\end{aligned}
$$

where $\Pi$ normalizes vectors to unit length, $\mathcal{R}_{\eta}$ rotates vectors by a random angle distributed uniformly in a range $(-\pi \eta ; \pi \eta]$, and the average is over the particles $j$ present in $\partial_{i}$, the disk of radius $r_{0}=1$ centered on $\mathbf{r}_{i}$. We checked that the results presented in the following are not sensitive to the value of $\alpha$, provided that $0<\alpha<1$. Therefore, in the following, we use only the numerically convenient value $\alpha=\frac{1}{2}$. With $\alpha$ fixed, the main parameters remain those of classic Vicsek-style models, the mean density of particles $\rho_{0}$, and the noise amplitude $\eta$.

We numerically determined the phase diagram of our model in the $\left(\rho_{0}, \eta\right)$ plane [Fig. 1(a)]. We find a single transition line from the disordered gas observed at strong noise and/or low density to a phase with global ordering of polarities, characterized, at finite system size, by a finite average value of the magnetization $M(t)=\left|\left\langle\mathbf{p}_{i}(t)\right\rangle_{i}\right|$. Contrary to the other known classes mentioned in the introduction, we do not see any sign of phase separation. The transition seems continuous, with only quasi-longrange order: the magnetization decreases algebraically with system size, $\langle M\rangle_{t} \sim L^{-\kappa(\eta)}$, with $\kappa$ increasing continuously with $\eta$ [Fig. 1(c)]. At strong-enough noise, a crossover to a fully disordered phase characterized by $\langle M\rangle_{t} \sim 1 / L$ is observed at large-enough sizes [Fig. 1(d)]. Like in all known orientationally ordered dry active matter phases, giant number fluctuations are present [Fig. 1(b)]: the variance $\left\langle\Delta N^{2}\right\rangle$ of the number of particles in a subsystem (a)

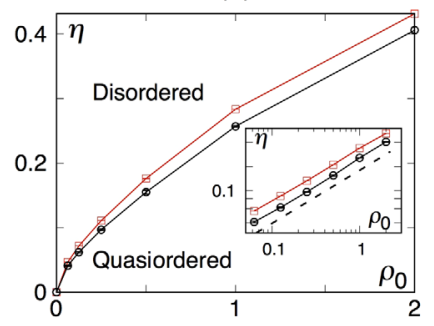

(c)
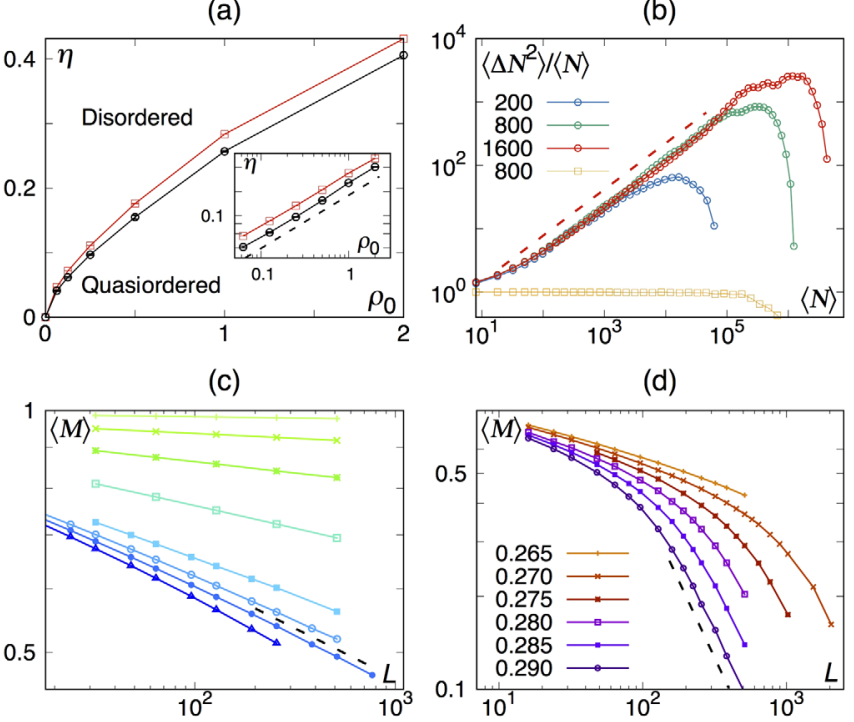

(d)

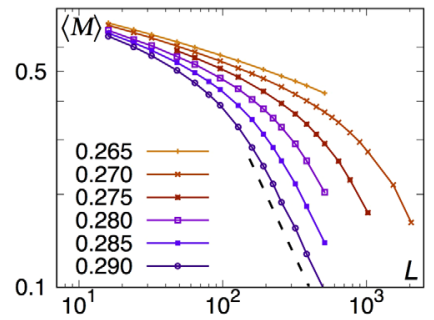

FIG. 1. (a) Phase diagram in the $\left(\rho_{0}, \eta\right)$ plane. The asymptotic order-disorder transition line is shown in black. The red curve reports the location of the susceptibility peak $\eta_{\chi}$ measured for $L=256$ (the error bars are smaller than the symbols). (Inset) The same data in logarithmic scales, with the dashed line marking slope 0.66. (b) Variance $\left\langle\Delta N^{2}\right\rangle$ over mean $\langle N\rangle$ of the number of particles present in subsystems in the quasiordered (the circles, $\eta=0.2$ ) and disordered (the squares, $\eta=0.5$ ) phases for various system sizes $L\left(\rho_{0}=2\right)$. The dashed line corresponds to $\zeta=1.73$. (c),(d) Averaged magnetization as a function of system size for several noise values in the quasiordered and disordered phases $\left(\rho_{0}=1\right)$. In the quasiordered state, $\langle M\rangle$ decays algebraically, with an $\eta$-dependent exponent $\kappa(\eta)$. The curves in (c) correspond to, from top to bottom, $\eta=0.05,0.1,0.15,0.2,0.24$, $0.25,0.255$, and 0.26 . In (c) $[(\mathrm{d})]$ the dashed black line marks the slope $-\frac{1}{8}(-1)$.

containing, on average, $\langle N\rangle$ particles scales faster than $\langle N\rangle$. We find that $\left\langle\Delta N^{2}\right\rangle \sim\langle N\rangle^{\zeta}$, with $\zeta=1.73(3)$, a value similar to those reported for the other classes $[22,25,36]$.

We now derive hydrodynamic equations for the Vicsekshake class from our microscopic model. Encouraged by its overall success in the other cases, we adopt the Boltzmann-Ginzburg-Landau approach [19,23,37]. We write two coupled Boltzmann equations for the single-body distributions $f_{ \pm}(\mathbf{x}, \theta, t)$ of + and - particles, i.e., those which, respectively, currently move along or against their polarity:

$\partial_{t} f_{ \pm} \pm \mathbf{e}(\theta) \cdot \boldsymbol{\nabla} f_{ \pm}=a\left(f_{\mp}-f_{ \pm}\right)+I_{\mathrm{dif}}\left[f_{ \pm}\right]+I_{\mathrm{col}}\left[f_{ \pm}, f_{\mp}\right]$

where $\mathbf{e}(\theta)$ in the material derivatives of $f_{ \pm}$is the unit vector along $\theta$ [53], $a$ is the exchange rate between the two subpopulations (akin to the microscopic reversal probability $\alpha$ ), and the self-diffusion and collisional integrals read 


$$
\begin{aligned}
I_{\mathrm{dif}}\left[f_{ \pm}\right]=- & f_{ \pm}+\int_{0}^{2 \pi} d \theta^{\prime} \int_{-\infty}^{\infty} d \sigma f_{ \pm}\left(\theta^{\prime}\right) P_{\eta}(\sigma) \delta_{2 \pi}\left(\theta-\theta^{\prime}-\sigma\right), \\
I_{\mathrm{col}}\left[f_{+}, f_{-}\right]= & \int_{0}^{2 \pi} d \theta_{1} \int_{0}^{2 \pi} d \theta_{2} \int_{-\infty}^{\infty} d \sigma P_{\eta}(\sigma) f_{+}\left(\theta_{1}\right) \\
& \times\left[f_{+}\left(\theta_{2}\right) K^{+}\left(\theta_{1}-\theta_{2}\right)+f_{-}\left(\theta_{2}\right) K^{-}\left(\theta_{1}-\theta_{2}\right)\right] \\
& \times\left\{\delta_{2 \pi}\left[\theta-\Psi\left(\theta_{1}, \theta_{2}\right)-\sigma\right]-\delta_{2 \pi}\left(\theta-\theta_{1}\right)\right\}
\end{aligned}
$$

where $P_{\eta}(\sigma)$ is the noise distribution of variance $\eta^{2}, \delta_{2 \pi}$ is the Dirac comb distribution of period $2 \pi$, and $\Psi\left(\theta_{1}, \theta_{2}\right)=$ $\arg \left[\mathbf{e}\left(\theta_{1}\right)+\mathbf{e}\left(\theta_{2}\right)\right]$ is the ferromagnetic alignment rule of polarities. The kernels $K^{ \pm}\left(\theta_{1}-\theta_{2}\right)=\left|\mathbf{e}\left(\theta_{1}\right) \mp \mathbf{e}\left(\theta_{2}\right)\right|$ are different and are used depending on whether or not the two colliding particles belong to the same population.

Hydrodynamic equations are derived from Eq. (3) by expanding the distributions in angular Fourier modes: $f_{ \pm}=\sum f_{k}^{ \pm} \exp (-i k \theta) /(2 \pi)$, and truncating and closing the resulting hierarchies in a controlled way. In the classic Vicsek model, the remaining hydrodynamic (or slow) fields correspond to the first two angular modes, i.e., density and polarity (or velocity). Here, these fields are the zeroth and first modes of the sum $f=f_{+}+f_{-}$, i.e., the density and polarity of the total population, while the velocity field, now distinct from polarity, is the first mode of the difference $g=f_{+}-f_{-}$. Rewriting the Boltzmann equations in terms of the $f$ and $g$ modes, we obtain

$$
\begin{aligned}
\partial_{t} f_{k} & +\frac{1}{2}\left(\nabla^{*} g_{k+1}+\nabla g_{k-1}\right) \\
= & \left(P_{k}-1\right) f_{k}+\sum_{q=-\infty}^{+\infty} A_{k, q} f_{q} f_{k-q}+B_{k, q} g_{q} g_{k-q}, \\
\partial_{t} g_{k} & +\frac{1}{2}\left(\nabla^{*} f_{k+1}+\nabla f_{k-1}\right) \\
= & \left(P_{k}-1-2 a\right) g_{k}+\sum_{q=-\infty}^{+\infty} C_{k, q} f_{q} g_{k-q},
\end{aligned}
$$

where the complex gradient $\nabla=\partial_{x}+i \partial_{y}, \quad P_{k}=$ $\int d \sigma P_{\eta}(\sigma) \exp (i k \sigma)$, and all other coefficients are listed in the Supplemental Material [54]. Setting $f_{1} \sim \varepsilon$ near the onset of polar order, Eqs. (6) and (7) impose the following scaling ansatz [37]:

$$
\begin{gathered}
\delta \rho=\rho-\rho_{0} \sim g_{0} \sim \varepsilon, \quad\left|f_{k}\right| \sim\left|g_{k}\right| \sim \varepsilon^{k} \quad \forall k>0, \\
\partial_{t} \sim \nabla \sim \varepsilon .
\end{gathered}
$$

At the first nontrivial order, $\varepsilon^{3}$, we get equations for $\rho$, $g_{0}, f_{1}, g_{1}, f_{2}$, and $g_{2}$. The last two fields can then be enslaved to the four remaining ones, yielding

$$
\begin{aligned}
\partial_{t} \rho= & -\operatorname{Re}\left(\nabla^{*} g_{1}\right), \\
\partial_{t} g_{0}= & -2 a g_{0}-\operatorname{Re}\left(\nabla^{*} f_{1}\right), \\
\partial_{t} f_{1}= & \left(\mu_{1}[\rho]-\xi\left|f_{1}\right|^{2}-\delta\left|g_{1}\right|^{2}\right) f_{1}+\Gamma \Delta f_{1} \\
& +\left(\gamma\left[g_{0}\right]-\beta f_{1}^{*} g_{1}\right) g_{1}-\frac{1}{2} \nabla g_{0}+\eta_{1} f_{1}^{*} \nabla g_{1} \\
& +\eta_{2} g_{1}^{*} \nabla f_{1}+\eta_{3} \nabla^{*}\left(f_{1} g_{1}\right),
\end{aligned}
$$

$$
\begin{aligned}
\partial_{t} g_{1}= & \left(\nu_{1}[\rho]-\tau\left|g_{1}\right|^{2}-\omega\left|f_{1}\right|^{2}\right) g_{1}+\lambda \Delta g_{1} \\
& +\left(\kappa\left[g_{0}\right]-\chi g_{1}^{*} f_{1}\right) f_{1}-\frac{1}{2} \nabla \rho+\sigma_{1} g_{1}^{*} \nabla g_{1} \\
& +\sigma_{2} f_{1}^{*} \nabla f_{1}+\sigma_{3} \nabla^{*} f_{1}^{2}+\sigma_{4} \nabla^{*} g_{1}^{2}
\end{aligned}
$$

where $\Delta=\nabla \nabla^{*}$ and all coefficients, expressed as functions of the microscopic parameters $\rho_{0}, \eta$, and $a$, are listed in the Supplemental Material [54], while their dependence on local density and $g_{0}$ has been made explicit. Equations (10)-(13) can be seen as two coupled Toner-Tu equations [14,55]. Note that density is advected not by the order field $f_{1}$ but by $g_{1}$, in strong contrast to the classic polar case. Since $\nu_{1}[\rho]<0$ and $\mu_{1}[\rho]$ can change sign, the transition, as expected, is given by $\mu_{1}\left[\rho_{0}\right]=0$, defining a line in the $\left(\rho_{0}, \eta\right)$ plane that goes to the origin as $\sqrt{\rho_{0}}$. Furthermore, since $\mu_{1}[\rho]$ does not depend on $a$, this line is insensitive to the reversal rate, in agreement with the microscopic model. When $\mu_{1}\left[\rho_{0}\right]<0$ the homogeneous disordered solution $\rho=\rho_{0}, f_{1}=g_{0}=$ $g_{1}=0$ is linearly stable, and it becomes unstable when $\mu_{1}\left[\rho_{0}\right]>0$. It is then replaced by the homogeneous ordered solution $\rho=\rho_{0}, g_{0}=g_{1}=0, f_{1}=\sqrt{\mu_{1}\left[\rho_{0}\right] / \xi}$. We studied its linear stability seminumerically (see the Supplemental Material [54] for details) and analytically in the long wavelength limit (not shown). Apart from a pocket of weak, spurious instability at small $a$ and low noises, it is essentially stable as soon as $\mu_{1}\left[\rho_{0}\right]>0$ [56].

The analysis above confirms, at the mean-field level, the absence of the generic instability leading to the phase separation scenario in other classes of dry, aligning active matter. Here, we have a single transition line separating polar order from the disordered phase. Order (field $f_{1}$ ) and density are advected by the auxiliary field $g_{1}$, and thus the mechanism proven by Toner and Tu to be responsible for the possibility of true long-range order is absent $[14,15]$. With fluctuations, polar order is only quasi-long-range, as in equilibrium. Our problem thus possesses many of the hallmarks of the $X Y$ model. We now investigate whether this extends to the nature of the transition, i.e., whether it is in the well-known Berezinskii-Kosterlitz-Thouless (BKT) universality class. The BKT transition is characterized by an essential divergence of the correlation length $\xi$ when approaching the critical point $\eta_{c}$ from the disorder side, together with the scaling of the susceptibility $\chi$ with $\xi[51,52,57]$, 


$$
\begin{aligned}
\log \xi & \sim\left(\eta-\eta_{c}\right)^{-\nu}, \\
\chi & \sim \xi \tilde{\gamma}, \quad \text { with } \quad \chi=L^{2}\left(\left\langle M^{2}\right\rangle-\langle M\rangle^{2}\right),
\end{aligned}
$$

with $\nu=\frac{1}{2}$ and $\tilde{\gamma}=\gamma / \nu=\frac{7}{4}$. At finite size $L, \chi$ exhibits a maximum $\chi_{\max }(L)$ located at $\eta_{\chi}(L)$. Increasing $L, \chi_{\max }$ diverges, and $\eta_{\chi}$ converges to $\eta_{c}$ like

$\chi_{\max }(L) \sim L^{\tilde{\gamma}}, \quad \eta_{\chi}(L)-\eta_{c} \sim[\log (L)-a]^{-1 / \nu}$.

We have measured the dependence of $\langle M\rangle$ and $\chi$ on $\eta$ for various system sizes, all at various global density values, but focusing most of our numerical effort on $\rho_{0}=1$. As shown in Fig. 2(a), the susceptibility peak $\chi_{\max }$ does diverge algebraically with an exponent $\tilde{\gamma}=1.755(6)$, in full agreement with the BKT-Ising value of $\frac{7}{4}$. The peak location $\eta_{\chi}$ is reasonably well fitted by Eq. (15) with $\nu=\frac{1}{2}$, yielding an estimate of the asymptotic threshold $\eta_{c}=0.247(2)$ [Fig. 2(b)].

We defined the correlation length $\xi$ as the crossover scale marking the beginning of the $1 / L$ decay in $\langle M\rangle(L)$ curves at a fixed $\eta$ (see the Supplemental Material [54] for details). The divergence of $\xi$ with decreasing values of $\eta$ is well fitted by Eq. (14) with $\nu=\frac{1}{2}$ [Fig. 2(c)]. This fit yields an estimate $\eta_{c}=0.244(2)$ that is (barely) compatible with (a)

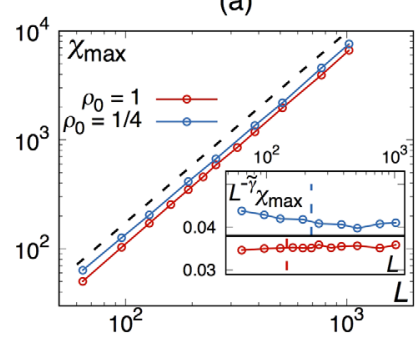

(c)

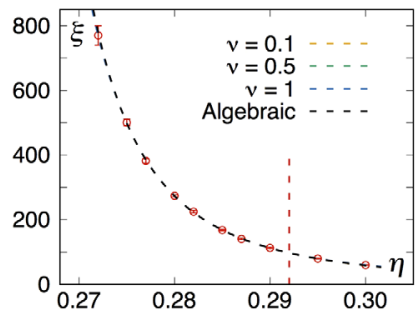

(b)

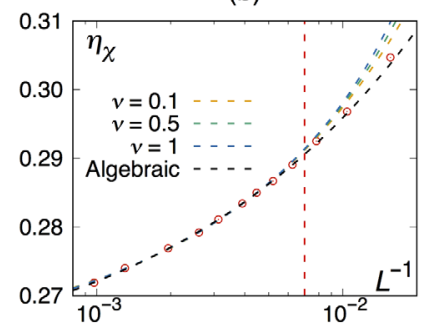

(d)

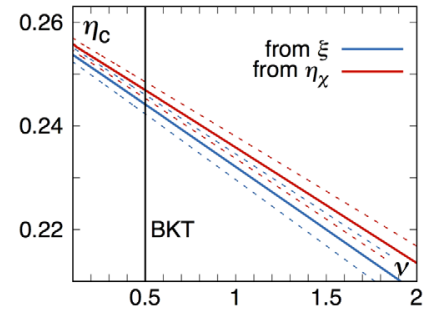

FIG. 2. (a) Susceptibility peak maximum $\chi_{\max }$ vs system size $L$. The dashed line has the slope 1.75. (Inset) The same data scaled with $L^{-\tilde{\gamma}}$, with $\tilde{\gamma}=1.75$. Here and in (b) and (c), the vertical dashed line delimits system sizes below which the scaling regime is not reached [these points are not used for the fits in (b), (c), and (d)]. (b) Position of $\chi_{\max }$ vs $L^{-1}$. The different lines are fits using Eq. (15) (BKT-like scaling) for several values of $\nu$ and Eq. (16) (algebraic scaling). (c) The same as (b) but for divergence of the correlation length with noise. (d) Asymptotic threshold $\eta_{c}$ obtained from fits of $\xi$ and $\eta_{\chi}$ with BKT-like scaling (14) and (15) varying the exponent $\nu$. The dashed lines represent the confidence intervals on $\eta_{c}$ given by the fits. that obtained from the susceptibility. However, for $0.244<\eta<0.247,\langle M\rangle$ decreases with an exponent $\kappa(\eta) \simeq 0.100(5)$ that is incompatible with the BKT value $\frac{1}{8}$ [see Fig. 1(c)]. Repeating the procedure for $\rho_{0}=\frac{1}{2}$ and 2 , we reach the same conclusion and find different values of $\kappa\left(\eta_{c}\right)$, respectively, 0.089(6) and 0.117(2).

Allowing now $\nu$ to vary in a range $[0.1,2]$, we find fits of the variations of $\eta_{\chi}$ and $\xi$ as convincing as for the BKT value $\nu=\frac{1}{2}$. Interestingly, the two independent estimates of $\eta_{c}$ then become closer to each other as $\nu \rightarrow 0$ [Fig. 2(d)]. This suggests an algebraic divergence for $\xi$ at threshold. We therefore redefine the $\nu$ exponent as that of a standard second-order phase transition:

$$
\xi \sim\left(\eta-\eta_{c}\right)^{-\nu}, \quad \eta_{\chi}(L)-\eta_{c} \sim L^{-1 / \nu} .
$$

Fitting our data accordingly, we obtain better fits for both $\xi$ and $\eta_{\chi}$ and, importantly, fully compatible threshold values at which, moreover, $\kappa\left(\eta_{c}\right) \simeq \frac{1}{8}$. Imposing a common value for the asymptotic threshold, both data sets give the same estimate of $\nu$, and we finally conclude that $\eta_{c}=0.257(1)$ with $\nu=2.4(1)$. From these values, we compute $\beta / \nu$ using a collapse of the magnetization curves (shown in the Supplemental Material [54]) and find a value fully compatible with $\beta / \nu=\frac{1}{8}$, which satisfies the hyperscaling relation $2 \beta / \nu+\gamma / \nu=d$, with $d=2$. Using data obtained at various global densities, we find the same estimates of $\gamma / \nu$ and $\beta / \nu$, although our estimate of $\nu$ shows some variation due to its sensitivity to the estimated value of $\eta_{c}$. The asymptotic threshold values thus obtained behave as $\eta_{c} \sim \rho_{0}^{h}$, with $h \sim 0.66$, a clear departure from the meanfield value $\frac{1}{2}$ [Fig. 1(a)].

Our numerical analysis leads us to conclude that the transition to polar order exhibited by our system is not of the BKT type. In the $X Y$ model, this transition is closely related to the (effective) Coulomb interaction between topological singularities that unbind and proliferate above the critical temperature [51,52]. Detecting these defects in simulations of our model is made very difficult, if not impossible, by the presence of strong density fluctuations. Indeed, the very existence of topologically constrained defects requires that order can be defined everywhere. Here, the local order is hard to measure in sparse regions, and even impossible to define if the local density is below the ordering threshold $\rho_{0}^{c}(\eta)$, the transitional density found by varying $\rho_{0}$ while keeping $\eta$ fixed. One can nevertheless study the fate of defects from carefully prepared initial configurations containing a \pm 1 pair. Running the model deep in the ordered phase, we observe that the positive defect expels particles from its core and is quickly transformed into a sparse, almost void region whose diameter grows like $\sqrt{t}$ [Fig. 3(c)]. After some time, this region has become sufficiently large so that it reaches the negative defect and the system eventually repairs itself [see Fig. 3(a) and the movie in the Supplemental 
(a)

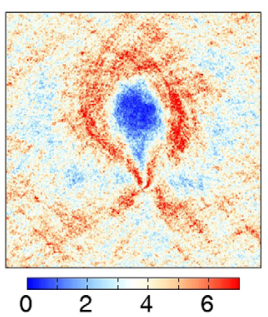

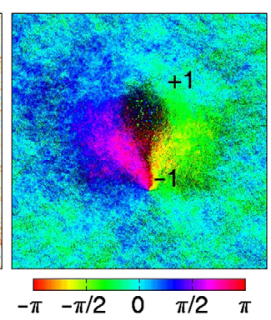

(b)

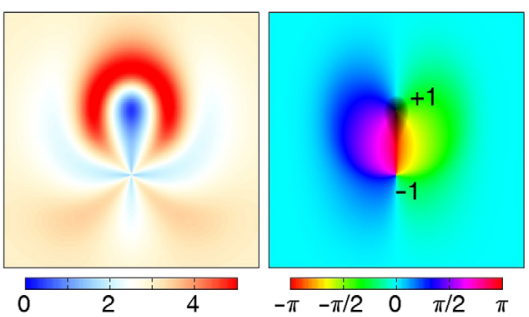

(c)

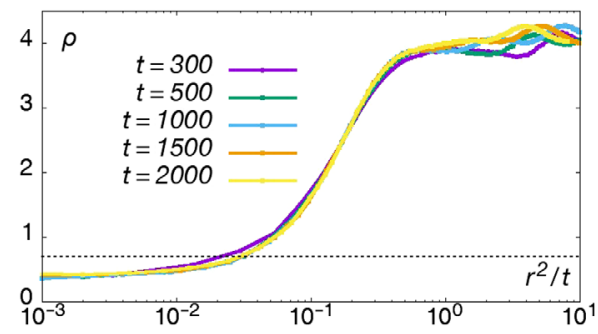

FIG. 3. (a),(b) Annihilation of an initially prepared pair of defects deep in the ordered phase. (a) Snapshots of density and polarity orientation fields from our microscopic model $\left(\rho_{0}=4, \eta=0.2, L^{2}=1024^{2}\right.$, and $\left.t=40000\right)$. (b) The same as (a) but from a simulation of hydrodynamic equations (10)-(13) $\left(\rho_{0}=3, \eta=0.4, a=1, L^{2}=256^{2}\right.$, and $\left.t=2500\right)$. (c) Time-rescaled radial density profiles around the +1 defect in microscopic simulations [the same parameters as in (a)]. The dashed line indicates the transitional density $\rho_{0}^{c}(\eta)$ corresponding to $\eta=0.2$.

Material [54]]. This mean-field behavior is also observed in simulations of the (deterministic) hydrodynamic equations [see Fig. 3(b) and the movie in the Supplemental Material [54]]. It is intrinsically related to the coupling between density and order and indicates that the very idea of topologically bound point defects is not relevant in our system. Closer to the transition, we, of course, expect fluctuations to play a major role, but this conclusion should still hold. This is the topic of ongoing work.

To summarize, we have shown that the collective behavior of active particles with velocity reversals that align ferromagnetically their polarities is different from that of other classes of dry, dilute, aligning active matter. This new class is characterized by the emergence of a phase with quasi-long-range polar order and anomalous number fluctuations. As in the $X Y$ model, scaling exponents vary continuously in this phase, but the transition point to order shows algebraic divergences governed by $\nu=2.4(1)$, not the essential singularity of the BKT class. This value does not seem to correspond to any known class. Nevertheless, the exponent ratios $\beta / \nu$ and $\gamma / \nu$ take the BKT-Ising values $\frac{1}{8}$ and $\frac{7}{4}$. These results constitute the first case where the phase separation scenario at play in most dry, aligning active matter systems is prevented "structurally".

We thank Francesco Ginelli, Cesare Nardini, and Julien Tailleur for the helpful discussions and their critical reading of our manuscript. This work is partially supported by ANR project Bactterns, FRM project Neisseria, and the National Natural Science Foundation of China (Grant No. 11635002 to X.-q. S. and H. C., Grants No. 11474210 and No. 11674236 to X.-q. S., Grants No. 11474155 and No. 11774147 to Y.-q. M.).

[1] S. Ramaswamy, The mechanics and statistics of active matter, Annu. Rev. Condens. Matter Phys. 1, 323 (2010).

[2] M. C. Marchetti, J.-F. Joanny, S. Ramaswamy, T. B. Liverpool, J. Prost, M. Rao, and R. A. Simha, Hydrodynamics of soft active matter, Rev. Mod. Phys. 85, 1143 (2013).
[3] A. Cavagna, A. Cimarelli, I. Giardina, G. Parisi, R. Santagati, F. Stefanini, and M. Viale, Scale-free correlations in starling flocks, Proc. Natl. Acad. Sci. U.S.A. 107, 11865 (2010).

[4] T. Vicsek and A. Zafeiris, Collective motion, Phys. Rep. 517, 71 (2012).

[5] H.-P. Zhang, A. Be'er, E.-L. Florin, and H. L. Swinney, Collective motion and density fluctuations in bacterial colonies, Proc. Natl. Acad. Sci. U.S.A. 107, 13626 (2010).

[6] F. Peruani, J. Starruß, V. Jakovljevic, L. Søgaard-Andersen, A. Deutsch, and M. Bär, Collective Motion and Nonequilibrium Cluster Formation in Colonies of Gliding Bacteria, Phys. Rev. Lett. 108, 098102 (2012).

[7] D. Nishiguchi, K. H. Nagai, H. Chaté, and M. Sano, Longrange nematic order and anomalous fluctuations in suspensions of swimming filamentous bacteria, Phys. Rev. E 95, 020601 (2017).

[8] Y. Sumino, K. H. Nagai, Y. Shitaka, D. Tanaka, K. Yoshikawa, H. Chaté, and K. Oiwa, Large-scale vortex lattice emerging from collectively moving microtubules, Nature (London) 483, 448 (2012).

[9] V. Narayan, S. Ramaswamy, and N. Menon, Long-lived giant number fluctuations in a swarming granular nematic, Science 317, 105 (2007).

[10] J. Deseigne, O. Dauchot, and H. Chaté, Collective Motion of Vibrated Polar Disks, Phys. Rev. Lett. 105, 098001 (2010).

[11] N. Kumar, H. Soni, S. Ramaswamy, and A. Sood, Flocking at a distance in active granular matter, Nat. Commun. 5, 4688 (2014).

[12] C. A. Weber, T. Hanke, J. Deseigne, S. Léonard, O. Dauchot, E. Frey, and H. Chaté, Long-Range Ordering of Vibrated Polar Disks, Phys. Rev. Lett. 110, 208001 (2013).

[13] T. Vicsek, A. Czirók, E. Ben-Jacob, I. Cohen, and O. Shochet, Novel Type of Phase Transition in a System of Self-Driven Particles, Phys. Rev. Lett. 75, 1226 (1995).

[14] J. Toner and Y. Tu, Long-Range Order in a Two-Dimensional Dynamical XY Model: How Birds Fly Together, Phys. Rev. Lett. 75, 4326 (1995).

[15] J. Toner and Y. Tu, Flocks, herds, and schools: A quantitative theory of flocking, Phys. Rev. E 58, 4828 (1998).

[16] S. Ramaswamy, R. A. Simha, and J. Toner, Active nematics on a substrate: Giant number fluctuations and long-time tails, Europhys. Lett. 62, 196 (2003). 
[17] G. Grégoire and H. Chaté, Onset of Collective and Cohesive Motion, Phys. Rev. Lett. 92, 025702 (2004).

[18] J. Toner, Y. Tu, and S. Ramaswamy, Hydrodynamics and phases of flocks, Ann. Phys. (Amsterdam) 318, 170 (2005).

[19] E. Bertin, M. Droz, and G. Grégoire, Boltzmann and hydrodynamic description for self-propelled particles, Phys. Rev. E 74, 022101 (2006).

[20] H. Chaté, F. Ginelli, and R. Montagne, Simple Model for Active Nematics: Quasi-Long-Range Order and Giant Fluctuations, Phys. Rev. Lett. 96, 180602 (2006).

[21] A. Baskaran and M. C. Marchetti, Enhanced Diffusion and Ordering of Self-Propelled Rods, Phys. Rev. Lett. 101, 268101 (2008).

[22] H. Chaté, F. Ginelli, G. Grégoire, and F. Raynaud, Collective motion of self-propelled particles interacting without cohesion, Phys. Rev. E 77, 046113 (2008).

[23] E. Bertin, M. Droz, and G. Grégoire, Hydrodynamic equations for self-propelled particles: Microscopic derivation and stability analysis, J. Phys. A 42, 445001 (2009).

[24] A. Baskaran and M. C. Marchetti, Nonequilibrium statistical mechanics of self-propelled hard rods, J. Stat. Mech. (2010) P04019.

[25] F. Ginelli, F. Peruani, M. Bär, and H. Chaté, Large-Scale Collective Properties of Self-Propelled Rods, Phys. Rev. Lett. 104, 184502 (2010).

[26] S. Mishra, R. A. Simha, and S. Ramaswamy, A dynamic renormalization group study of active nematics, J. Stat. Mech. (2010) P02003.

[27] T. Ihle, Kinetic theory of flocking: Derivation of hydrodynamic equations, Phys. Rev. E 83, 030901 (2011).

[28] F. D. C. Farrell, M. C. Marchetti, D. Marenduzzo, and J. Tailleur, Pattern Formation in Self-Propelled Particles with Density-Dependent Motility, Phys. Rev. Lett. 108, 248101 (2012).

[29] R. Grossmann, L. Schimansky-Geier, and P. Romanczuk, Active Brownian particles with velocity-alignment and active fluctuations, New J. Phys. 14, 073033 (2012).

[30] A. Peshkov, I. S. Aranson, E. Bertin, H. Chaté, and F. Ginelli, Nonlinear Field Equations for Aligning SelfPropelled Rods, Phys. Rev. Lett. 109, 268701 (2012).

[31] P. Romanczuk and L. Schimansky-Geier, Mean-field theory of collective motion due to velocity alignment, Ecol. Complexity 10, 83 (2012).

[32] J. Toner, Reanalysis of the hydrodynamic theory of fluid, polar-ordered flocks, Phys. Rev. E 86, 031918 (2012).

[33] E. Bertin, H. Chaté, F. Ginelli, S. Mishra, A. Peshkov, and S. Ramaswamy, Mesoscopic theory for fluctuating active nematics, New J. Phys. 15, 085032 (2013).

[34] A. P. Solon and J. Tailleur, Revisiting the Flocking Transition Using Active Spins, Phys. Rev. Lett. 111, 078101 (2013).

[35] J.-B. Caussin, A. Solon, A. Peshkov, H. Chaté, T. Dauxois, J. Tailleur, V. Vitelli, and D. Bartolo, Emergent Spatial Structures in Flocking Models: A Dynamical System Insight, Phys. Rev. Lett. 112, 148102 (2014).

[36] S. Ngo, A. Peshkov, I. S. Aranson, E. Bertin, F. Ginelli, and H. Chaté, Large-Scale Chaos and Fluctuations in Active Nematics, Phys. Rev. Lett. 113, 038302 (2014).

[37] A. Peshkov, E. Bertin, F. Ginelli, and H. Chaté, BoltzmannGinzburg-Landau approach for continuous descriptions of generic Vicsek-like models, Eur. Phys. J. Spec. Top. 223, 1315 (2014).

[38] E. Putzig and A. Baskaran, Phase separation and emergent structures in an active nematic fluid, Phys. Rev. E 90, 042304 (2014).

[39] F. Thüroff, C. A. Weber, and E. Frey, Numerical Treatment of the Boltzmann Equation for Self-Propelled Particle Systems, Phys. Rev. X 4, 041030 (2014).

[40] A. P. Solon, H. Chaté, and J. Tailleur, From Phase to Microphase Separation in Flocking Models: The Essential Role of Nonequilibrium Fluctuations, Phys. Rev. Lett. 114, 068101 (2015).

[41] R. Großmann, F. Peruani, and M. Bär, Mesoscale pattern formation of self-propelled rods with velocity reversal, Phys. Rev. E 94, 050602 (2016).

[42] S. Shankar, S. Ramaswamy, and M. C. Marchetti, Lownoise phase of a two-dimensional active nematic system, Phys. Rev. E 97, 012707 (2018).

[43] F. Ginelli and H. Chaté, Relevance of Metric-Free Interactions in Flocking Phenomena, Phys. Rev. Lett. 105, 168103 (2010).

[44] A. Peshkov, S. Ngo, E. Bertin, H. Chaté, and F. Ginelli, Continuous Theory of Active Matter Systems with MetricFree Interactions, Phys. Rev. Lett. 109, 098101 (2012).

[45] L. Chen, J. Toner, and C. F. Lee, Critical phenomenon of the order-disorder transition in incompressible active fluids, New J. Phys. 17, 042002 (2015).

[46] Of course, one can also render the density field "nonhydrodynamic" [J. Toner, Birth, Death, and Flight: A Theory of Malthusian Flocks, Phys. Rev. Lett. 108, 088102 (2012)] or entirely eliminate space [M. Aldana and $\mathrm{C}$. Huepe, Phase transitions in self-driven many-particle systems and related non-equilibrium models: A network approach, J. Stat. Phys. 112, 135 (2003)], but the critical behavior should then be trivial.

[47] A. P. Solon, J.-B. Caussin, D. Bartolo, H. Chaté, and J. Tailleur, Pattern formation in flocking models: A hydrodynamic description, Phys. Rev. E 92, 062111 (2015).

[48] The ferromagnetic alignment is then destroyed by the stochastic reversals.

[49] An experimental realization could consist of vertically shaken granular disks, such as in Ref. [10], but could be endowed with two identical legs and carrying magnets. The symmetric legs would induce apolar motion with reversals. The magnets could dominate the interactions and lead to ferromagnetic alignment.

[50] V. Berezinskii, Destruction of long-range order in onedimensional and two-dimensional systems having a continuous symmetry group I. Classical systems, Sov. Phys. JETP 32, 493 (1971), http://www.jetp.ac.ru/cgi-bin/e/index/ $\mathrm{e} / 32 / 3 / \mathrm{p} 493$ ? $\mathrm{a}=$ list

[51] J. M. Kosterlitz and D. J. Thouless, Ordering, metastability and phase transitions in two-dimensional systems, J. Phys. C 6, 1181 (1973).

[52] J. Kosterlitz, The critical properties of the two-dimensional xy model, J. Phys. C 7, 1046 (1974).

[53] The speed $v_{0}$ has been set to unity without loss of generality.

[54] See Supplemental Material at http://link.aps.org/ supplemental/10.1103/PhysRevLett.120.258002 for (i) Coefficients of the finite reversal rate kinetic and hydrodynamic 
equations; (ii) Derivation of hydrodynamic equations in the large reversal rate limit; (iii) Linear stability of the homogeneous ordered state; (iv) Numerical methods and details; (v) Movies.

[55] The $g$ field equations both have a negative linear coefficient, regardless of density, noise, and reversal rate values. In the fast reversal limit, these fields can therefore be enslaved to $\rho$ and $f_{1}$. In the Supplemental Material [54], we derive equations directly in this limit and show that they behave similarly to the full equations.
[56] A small region of weak instability is present deep in the ordered phase for a small reversal rate $a$. It quickly disappears upon increasing $a$. Furthermore, we found that no such residual instability exists at the kinetic level, i.e., when considering Eqs. (6) and (7) with a large number of modes. We conclude that it is spurious (see the Supplemental Material [54] for details).

[57] R. Gupta, J. DeLapp, G. G. Batrouni, G. C. Fox, C. F. Baillie, and J. Apostolakis, Phase Transition in the 2D XY Model, Phys. Rev. Lett. 61, 1996 (1988). 